\title{
Shining a Light on the Mechanisms of Sleep for Memory Consolidation
}

\author{
Michelle A. Frazer ${ }^{1} \cdot$ Yesenia Cabrera $^{1} \cdot$ Rockelle S. Guthrie ${ }^{2} \cdot$ Gina R. Poe $^{3}$ (D)
}

Accepted: 23 March 2021 / Published online: 15 July 2021

(C) The Author(s) 2021

\begin{abstract}
Purpose of review This paper reviews all optogenetic studies that directly test various sleep states, traits, and circuit-level activity profiles for the consolidation of different learning tasks.

Recent findings Inhibiting or exciting neurons involved either in the production of sleep states or in the encoding and consolidation of memories reveals sleep states and traits that are essential for memory. REM sleep, NREM sleep, and the N2 transition to REM (characterized by sleep spindles) are integral to memory consolidation. Neural activity during sharp-wave ripples, slow oscillations, theta waves, and spindles are the mediators of this process.

Summary These studies lend strong support to the hypothesis that sleep is essential to the consolidation of memories from the hippocampus and the consolidation of motor learning which does not necessarily involve the hippocampus. Future research can further probe the types of memory dependent on sleep-related traits and on the neurotransmitters and neuromodulators required.
\end{abstract}

Keywords Theta $\cdot$ Sharp-wave ripples $\cdot$ Delta $\cdot$ Slow oscillations $\cdot$ Memory $\cdot$ LTP $\cdot$ Depotentiation $\cdot$ Timing $\cdot$ Rats $\cdot$ Mice . Optogenetics $\cdot$ Consolidation $\cdot$ Hippocampus $\cdot$ Motor learning $\cdot$ Spatial learning $\cdot$ Spindles $\cdot$ Replay

\section{Introduction}

Sleep is a period of heightened vulnerability for an organisman extended stretch of time during which an animal is oblivious to its surroundings, cut off from environmental cues that would alert it to a potential food source, an approaching mate, or an impending danger. And yet, despite all of the potential survival-related drawbacks that such a state entails, every animal sleeps. From drosophila to laboratory rats to humans, evolution has conserved this unguarded state across time. Clearly, the function of sleep is important enough to outweigh the

This article is part of the Topical Collection on Sleep and Learning

Gina R. Poe

ginapoe@ucla.edu

1 Brain Research Institute Neuroscience Interdepartmental Program, University of California, Los Angeles, Los Angeles, CA, USA

2 Department of Integrative Biology and Physiology, Molecular, Cellular, and Integrative Physiology Program, Program, University of California, Los Angeles, CA 90095, USA

3 The Department of Integrative Biology and Physiology, and Psychiatry and Biobehavioral Sciences, University of California, Los Angeles, 610 Charles E. Young Dr. E, Los Angeles, CA 90095, USA danger of being in such an unguarded state. Researchers have proposed a range of potential roles for each sleep state, including recovery from oxidative stress in the brain [1,2], energy conservation [3], a period of heightened protein synthesis [4, 5], and the stimulation of a sleeping brain to facilitate transitions into waking [6]. A somewhat more controversial proposition, however, is that sleep is an important stage of time for the consolidation of memories created during waking.

Sleep is divided into multiple stages (Fig. 1a); each of which is identified by stereotyped behavior, electrical activity, and neurochemical composition. The broadest distinction splits sleep into two stages, rapid eye movement (REM) and non REM (NREM) sleep. REM is characterized by muscle atonia, desynchronized waking-like EEG activity, and a 5-10 $\mathrm{Hz}$ hippocampal theta rhythm. NREM sleep displays $0-4 \mathrm{~Hz}$ slow oscillation / delta activity, accompanied by fast sharp-wave ripple (SWR) events in the hippocampus. Determining the role of each sleep stage and its signatures in the consolidation process is an area of intense investigation within the field of sleep research.

Experimentalists have been investigating the role of sleep in learning and memory storage for years, finding that depriving animals of normal sleep nearly always results in adverse outcomes in their ability to store and retain new information. For example, early studies of REM deprivation in rodents 
a

\section{REM

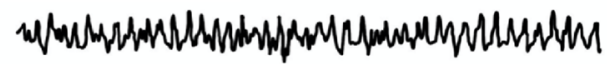

SWS

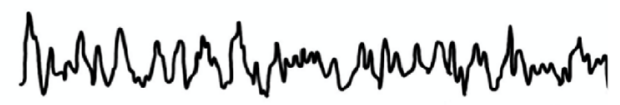

TR

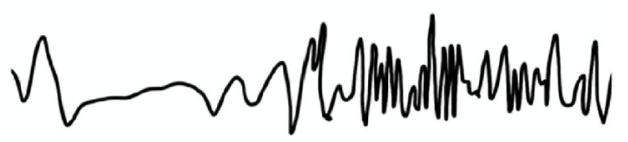

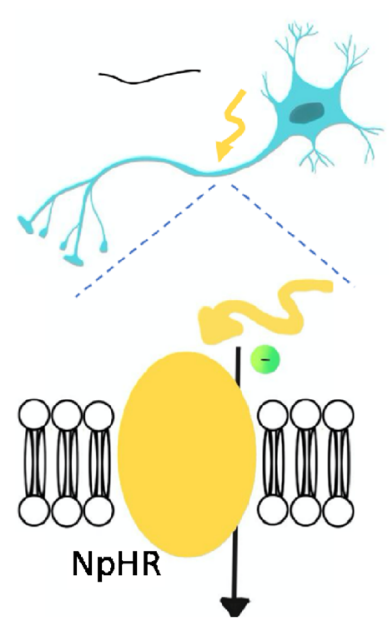

C

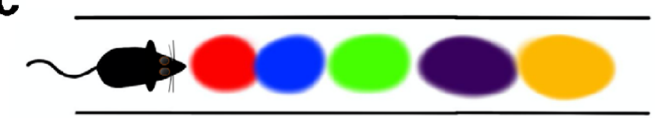

d

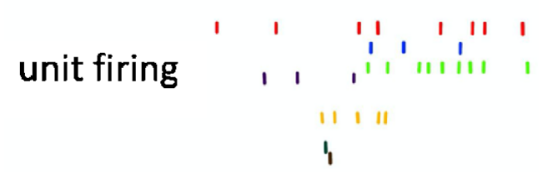

ripple event

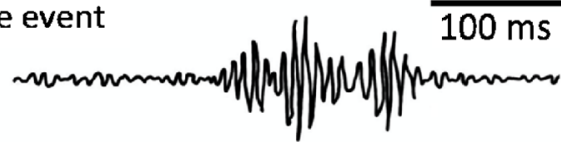

Fig. 1 A) Sample traces of rodent hippocampal EEG during sleep. Top: the $5-10 \mathrm{~Hz}$ theta of rapid eye movement (REM) sleep. Middle: Characteristic 2-4 Hz delta rhthym of slow wave sleep. Bottom: Examples of 10-14 Hz sigma in the form of sleep spindles that occur in brief periods of transition to REM (TR) sleep. B) Light-activated channels inserted into the neuronal membrane regulating activity. Stimulating channels such as ChR2 allow for the influx of cations when activated via blue light, resulting in membrane depolarization and action potential generation. To inhibit neural activity, light-triggered ion pumps are typically used, such as NpHR, which pumps in CI- ions to hyperpolarize the membrane. C). As an animal (left, from above) moves along its path, place cells (represented by different colors) encode the spatial context. D) An example of hippocampal replay during SWS. During SWS, ripple events in the hippocampus are generated by unit, firing of different place cells that at high frequencies, often in the same order as places encountered during waking.

showed decreased performance on hippocampal-dependent working memory tasks, especially contextual/spatial mazes that require established cognitive maps of the animals' environment [7-10]. Physiological studies indicate that REM deprivation results in a loss of excitability in the rodent hippocampus $[11,12]$ and reduces long-term potentiation (LTP) [13]. Accumulated evidence demonstrates that NREM sleep is beneficial for declarative memory in humans and may provide a time for communication between disparate brain areas, particularly the hippocampus and prefrontal cortex through the generation of sleep spindles, which are correlated to memory recall $[14,15]$. Conversely, REM sleep may be an important period for learning procedural tasks [16, 17].

While the body of evidence supporting an important role for sleep in memory consolidation grows, a number of critiques have plagued the field since its inception and proven themselves difficult to fully resolve. Limitations in the specificity of past methods as well as incongruous and conflicting study results have led some prominent sleep researchers to altogether reject any role for sleep in memory processing, instead viewing sleep solely through the lens of homeostatic regulation and systemic physiological function $[6,18,19]$. New techniques such as optogenetics enable researchers to target light-activated ion channels to control the activity of select brain areas, neural populations, or specific memory traces (Fig. 1b). Using light-activated channels such as channelrhodopsin (ChR2) and halorhodopsin (NpHR) allows for precise spatial and temporal neural activation or inhibition in order to tease apart the function of specific structures and cell populations [20]. Modulating neurons at such a granular level during sleep can tell us how activity outside of waking encoding (offline) shapes the connections and circuits of a memory ensemble. In this review, we aim to look at the ways in which optogenetics has allowed us to more specifically delineate the role of sleep in the consolidation of waking experiences (Table 1). We find that optogenetic techniques enable the modulation of various facets of sleep, while avoiding many of the confounding variables obscuring the results of earlier studies.

\section{Stress Is Not the Culprit: Sleep Feature Disruption Using Optogenetics Causes Learning and Memory Deficiencies Without the Stress of Behavioral Sleep Deprivation Methods}

One of the biggest critiques of early sleep studies was the difficulty in dissociating sleep loss from the stress induced by methods of sleep deprivation as playing the causal role in subsequent learning deficits. Methods to disrupt REM sleep can result in hypothalamic-pituitary-adrenal (HPA) axis activation and increased cortisol levels, which have been shown to negatively impact hippocampal dependent memory consolidation [36-38]. These methods led researchers to argue that 


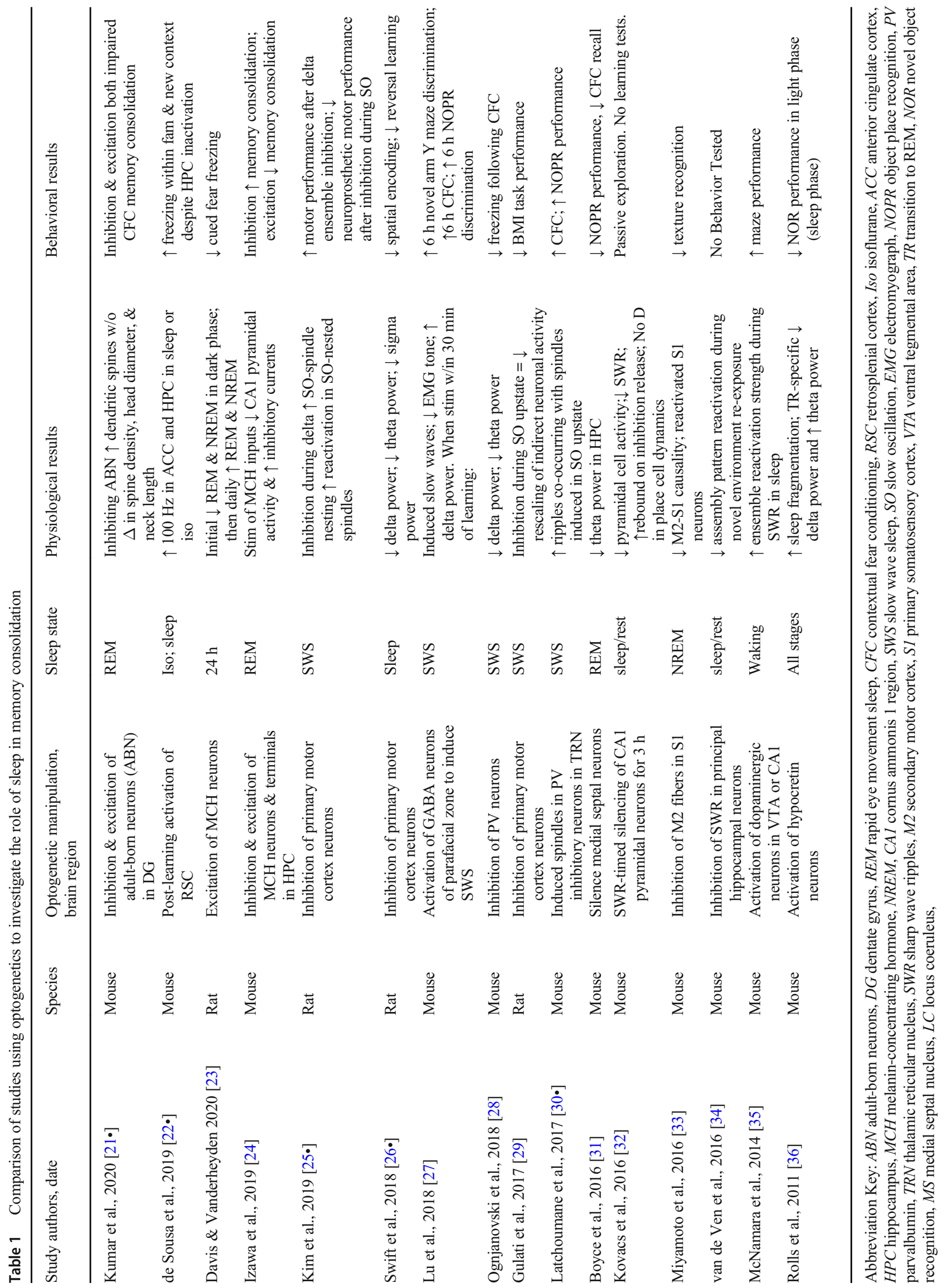


the behavioral deficits seen were a result of impaired hippocampal function due to stress rather than sleep loss. Indeed, stress does impair hippocampal neurogenesis just as chronic sleep disruption and sleep deprivation does, and perhaps the two mechanisms are the same [37, 38]. With the use of optogenetics, the studies discussed herein target specific sleep stages to modulate sleep features in a manner that does not introduce stress as a confounding variable.

One set of experiments used an optogenetic protocol to fragment sleep without inducing stress in the animal [36]. By selectively expressing $\mathrm{ChR} 2$, an excitatory optogenetic ion channel (Fig. 1b), in hypocretin/orexin (Hcrt) neurons in the lateral hypothalamus ( $\mathrm{LH})$, investigators were able to decrease latency to arousal in both REM and NREM, resulting in fragmented sleep periods. Stimulation of the LH at $60 \mathrm{~s}$ intervals with blue light resulted in Hrct neurons firing action potentials and was enough to fragment sleep, as measured by microarousal periods. Overall sleep amount, quality, and composition of sleep was unchanged, and no evidence of sleep debt occurred following the stimulation protocol, indicating that only the continuity of sleep was disrupted. Recent research suggests that this arousal effect might be mediated through extensive projections to the locus coeruleus (LC), an important structure for sleep and arousal regulation [39-41]. To ensure that the animal was not under stress due to stimulation, investigators tested cortisol levels in the plasma of both ChR2 and control mice following the 4-h stimulation protocol, finding no difference between stimulated and unstimulated animals. Similarly, animals that underwent the optogenetic stimulation did not show increased anxiety behavior in an open-field maze. Taken together, these findings show that the optogenetically induced sleep fragmentation protocol did not increase physiological markers of stress or behavioral indications of increased anxiety.

The Hcrt stimulation-induced sleep disruption paradigm allowed researchers to directly test the effect of discontinuous sleep on learning and memory. Using the novel object recognition (NOR) task as a measure of hippocampal- and sleepdependent memory [42-44], they ran animals through the optogenetic stimulation protocol during sleep in the crucial $4 \mathrm{~h}$ window during the light period immediately following training on the task [9]. In animals with Hcrt neurons expressing ChR2, the blue light stimulation resulted in a significant impairment in NOR performance as compared to control animals. Importantly, this effect was only seen when stimulation occurred in the light period following task acquisition, not in the dark period hours later (outside the critical consolidation window), highlighting the role of Hcrt neurons in sleepdependent consolidation rather than a result of aberrant Hcrt activity alone. Additionally, this effect was abolished when stimulation intervals were increased to $120 \mathrm{~s}$ apart, indicating that there is a minimum length (> $60 \mathrm{~s}$ ) of sleep "quanta" necessary for adequate memory consolidation in the rodent. Another study found that 60 -s long inductions of SWS repeatedly for $30 \mathrm{~min}$ within $30 \mathrm{~min}$ of learning stabilized the memory such that discrimination was maintained for as long as $6 \mathrm{~h}$ after object place exposure and novel arm Y maze task [27]. They produced SWS by optogenetically stimulating the GABAergic neurons in the parafacial zone, thereby increasing delta and reducing EMG activity as is consistent with the induction of SWS. The 60-s long inductions of SWS also stabilized contextual fear memory and improved context discrimination in these animals.

It would be interesting to know what that minimum undisturbed sleep quanta for memory stabilization and consolidation is in the human. Even as few as 5 airway obstructionrelated arousals per hour (equating to once every $12 \mathrm{~min}$ ) is associated with memory deficits in humans [45].

The ability to separate the effects of sleep deprivation from the methods used to sleep deprive and the negative effects they can cause allows research to move toward understanding the role that each component of sleep plays in the memory consolidation process. Work in our lab has used optogenetics to change baseline activity in neuromodulatory systems during sleep without disrupting overall time spent in sleep or sleep architecture itself [26•]. Unlike the Hcrt study, we did not induce arousals from sleep, yet found similar memory consolidation deficits related to changes in the electrophysiological features of each sleep state. We optogenetically modulated the locus coeruleus (LC) activity during sleep, and provided correlational evidence for the role various sleep signatures play in sleep-dependent consolidation. Typically, the LC quiets during NREM periods and is silenced during REM sleep [46]. Maintaining LC activity at waking levels throughout sleep after learning resulted in a decrease in the 1$4 \mathrm{~Hz}$ delta band and in the $10-14 \mathrm{~Hz}$ sigma band corresponding to sleep spindles. Longer spindles, which increased in control learning animals, were nearly eliminated when LC activity was maintained during sleep. In REM sleep, theta power was significantly decreased during LC stimulation. These changes were correlated with poor performance in incorporating changes made in a spatial memory task. Greater reductions in delta and theta band power were directly related to an increase in switching away from reliance on the hippocampal map. Similarly, reductions in spindle-ripple coupling predicted confusion between the original and modified food positions on the maze. The ability to adjust the frequency of LC activation with optogenetics during sleep allowed us to keep normal sleep bout lengths, yet still observe changes in memory consolidation.

The fact that changing the underlying physiology of sleep without disrupting other aspects of sleep affects memory consolidation suggests that the brain activity associated with each sleep state, and the particular neurochemical composition associated with each phase of sleep, is critical in supporting memory consolidation. Additional studies have found that 
optogenetic modulation of neuronal subtypes during sleep can similarly disrupt memory consolidation. Researchers investigating the inhibition of parvalbumin interneurons $(\mathrm{PV}+)$ during NREM sleep found that this intervention abolished the typical post-learning sleep increase in delta and theta power in the hippocampus [28]. In another group of animals that were sleep deprived, a similar decrease in delta and theta power was observed. These delta and theta power decreases were concurrent with disrupted contextual fear memory in both groups. However, activating hippocampal parvalbumin interneurons rhythmically at theta frequency during sleep deprivation stabilized CA1 population activity measured during subsequent sleep and rescued appropriate post-sleep hippocampus-dependent fear behavior. These findings suggest the necessity of sleep at least partially lies in the population activity engendered during the state and provides evidence that this activity directs the consolidation of memories.

\section{Optogenetics Allow Us to Target and Modulate Specific Sleep Signatures, to Test Their Causal Relationship to Learning and Memory}

\section{NREM Sleep}

The phenomenon of hippocampal replay present in NREM sleep has long intrigued sleep researchers as a possible mechanism by which labile memory traces in the hippocampus are stabilized and sent to the neocortex for long-term storage. Replay consists of cells active during waking task acquisition (Fig. 1c) being reactivated during sleep (Fig. 1d) with conserved temporal order. Ripples (Fig. 1d) take place in the CA1 region of the hippocampus indicating fast, synchronous excitatory inputs from the principal cells of the CA3. Replay of waking sequences occurs at the peak of ripples [47, 48]. Recruitment into ripple activity is at least in part associated with the strength of connections between cells formed during wakefulness - cells that are significantly more likely to fire together during waking are more likely to fire together during ripple associated replay events $[47,49]$. Thus cell participation in ripple events is heavily biased by waking patterns of activity. These findings served as the basis for the theory that SWR and replay activity therein are mechanisms for strengthening and storing memories.

Indeed, when SWR activity is electrically disrupted during sleep following acquisition of a new hippocampus-dependent task, a significant impairment is observed in an animal's ability to perform those tasks, likely due to aberrant consolidation [50, 51]. Whether this performance impairment was due to the loss of SWRs specifically, or simply the disruption of hippocampal population activity due to the electrical stimulation, was not testable until optogenetics became a common technique.
Studies utilizing optogenetics found that selectively inhibiting pyramidal cells of the CA1 once SWRs were detected resulted in a significant decrease in an animal's ability to reinstate assembly activity representing a novel environment [34]. Intriguingly, inhibiting SWRs did not result in a change in assembly activity representing already consolidated familiar environments, suggesting the necessity of hippocampal SWR activity for consolidating new information. In addition, novel assemblies that stabilized more rapidly than others during waking acquisition were also unaffected by the disruption of offline reactivation, perhaps because their original online activity was enough to result in synaptic stabilization, obviating the necessity of consolidation. These findings support the hypothesis that offline activity is important for resolving unfinished business, strengthening weak connections made during waking.

A second study using a similar paradigm in which principal CA1 cells express an inhibitory optogenetic ion channel investigated the effect of SWR suppression during the $3 \mathrm{~h}$ following passive exploration of a novel open field. They explored the ability of hippocampal place cells to subsequently encode the same open field environment after inhibiting pyramidal activity for $500 \mathrm{~ms}$ triggered after the detection of the ripple. No explicit learning task was given for the animals in the environment. Surprisingly, no significant differences were found between the place cell stability, coherence, or likelihood of remapping in the novel environment between animals with and without CA1 pyramidal activity during spontaneous SWR in this offline period [32]. Given the task impairment seen in Girardeau et al. [50], as well as the change in assembly activity in novel environments of the van de Ven [34] paper, one might conclude that disrupting the place cell code during the consolidation of novel memories underlies the spatial memory deficits seen following SWR inhibition. On the surface, this conclusion is not born out by Kovacs et al. [32]. However, they did observe a post-inhibition rebound in pyramidal cell activation that appeared to be coincident with strong local field potential (LFP) depolarization and, in their example, occurred in the ripple frequency. The apparent rebound depolarization-coupled ripple activity could itself have stabilized the memory code. Also, the authors point out that passive exploration may be relatively resistant to memory disruption by SWR inhibition as compared with active learning. For instance, a task in which learning is involved (and tested), such as an appetitive maze, should require the encoding and association of more information than is required when wandering in an open field. Another possibility is that ripples are involved primarily in the transmission of information to longterm cortical storage, and their disruption does not have an effect on spatial map stabilization within the hippocampus. Future experiments should be able to distinguish between these possibilities. 


\section{REM Sleep}

The question of whether REM sleep has a role in learning and memory has been particularly controversial $[6,18]$. In rodents, early sleep deprivation studies indicated that REM sleep is necessary for proper memory formation; however, as discussed above, the methods available at the time came with numerous caveats, leaving some sleep researchers skeptical of whether REM deprivation was truly causing memory impairments. Similar to our ability to optogenetically disrupt sleep as a whole, we now can design experiments selectively targeting REM sleep independently to isolate its role in memory processing without causing stress to the animal or affecting other sleep states. What we have learned about REM sleep thus far suggests that it serves several key functions in consolidation.

\section{REM Sleep Is for Forgetting}

REM sleep appears to be a time during which synapses are pruned, creating circuits with more sparse and more specific firing. Recent studies in mice show that the pruning of dendritic spines in the motor cortex (M1) during REM sleep is coincident with the improvement on a rotarod task $[52,53]$. Similarly, the firing of adult-born neurons (ABNs) in the dentate gyrus becomes more sparse in response to contextual fear conditioning (CFC) following a period of REM sleep [21•]. These data provide evidence that one of the functions of REM sleep is to pare down excessive activity following learning, and suggests a role for REM sleep in "forgetting" or weakening memories.

Studies of melanin-concentrating hormone $(\mathrm{MCH})$ neurons in the hypothalamus provide further evidence for the function of REM in forgetting [24]. Optogenetically inhibiting REMactive $\mathrm{MCH}$ neurons during REM periods following the learning phase of a novel object recognition (NOR) task improves the animals' ability to discriminate between new and familiar objects. The converse was also true-when $\mathrm{MCH}$ cells were optogenetically activated during REM periods, the animals' ability to discriminate between novel and familiar objects was diminished, as was their performance in the second task: contextual memory following fear conditioning. As this study also found that REM periods normally involve $\mathrm{MCH}$ cell activity, REM sleep appears to play a role in forgetting. It may be that the normal $\mathrm{MCH}$ activity during REM serves the function of schematic integration and extraction of gist, losing details as memories are moved to long-term storage or schematized.

\section{REM Sleep Is for Remembering}

To complicate matters further, however, evidence also exists that REM sleep promotes consolidation as well. The inhibition of theta activity by silencing medial septal GABAergic projections to the hippocampus during REM sleep significantly disrupted consolidation of CFC and novel object place recognition (NOPR) tasks [31]. This result raises the possibility that REM sleep is a time for both strengthening and weakening synapses due to the unique electrophysiological and neurochemical properties of the hippocampus in REM sleep. Hippocampal theta activity during REM sleep segregates the population activity into temporal cycles [54-57], which may be key to allowing both synaptic strengthening and weakening during this period of time. Previous studies from our lab show that spatial representations fire differently in relation to theta phase based on their novelty or familiarity [58]. New place cells fire at peak population activity, resulting in synaptic strengthening, while place cells encoding familiar spaces fire closer to the low point of activity, likely weakening the associated synapses through the principles of heterosynaptic depotentiation [58]. In addition to the theta rhythm, the neurochemical composition of the hippocampus during REM sleep, particularly the complete lack of noradrenaline and high levels of acetylcholine, could make it an optimal time for restructuring synaptic connections and integrating new information into old memory schema $[25 \cdot, 59]$.

\section{Population Activity and Timing Is Key in Sleep- Dependent Consolidation}

Through the temporal precision afforded by optogenetic techniques, sleep researchers have been able to tease apart the function of various sleep signatures and oscillatory activity. What we are finding suggests that the effect that a particular sleep stage or signature has on a neural ensemble is highly dependent on the population activity surrounding the ensemble. We have a great deal of evidence that reactivation of an ensemble during sleep strengthens the circuitry involved [47, 48]; however, it was initially unclear if this strengthening was simply a result of activity-dependent plasticity that could occur during any brain state. Furthermore, activity within sleep stages must be tightly temporally regulated in order to properly consolidate information. Studies suggest that the timing of action potentials of cells in an ensemble in relation to oscillatory population activity is critical to whether the connections of a memory trace will be strengthened or weakened. In a series of studies utilizing a brain-machine interface task in rats, researchers determined that whether an ensemble reactivated in closer proximity to a slow oscillation (SO) or to a delta wave had an effect on whether the memory was consolidated or weakened [29]. Ultimately, they found that perturbing spiking activity during the upstates of SOs led to an impairment of memory consolidation, whereas disrupting spiking during the upstate of a delta wave actually boosted sleep-dependent memory consolidation. Further exploration suggests that one underlying mechanism of the changes in consolidation is dependent on nesting of spindles within SOs or delta waves. SOspindle coupling appears to preserve memory reactivation, as 
reactivation strength and duration increased with $\mathrm{SO}$-spindle coupling, and changes to SO-spindle coupling correlated with task performance. Interestingly, when researchers optogenetically perturbed SO spiking activity, a rapid reduction in reactivation strength followed. The converse was true when delta activity was disrupted, resulting in significantly stronger and longer-lasting reactivation, supporting the idea that inhibiting delta waves increased the strength of a memory. Ultimately, inhibiting SO activity abolished any significant rescaling of the neural network involved in the task used, resulting in impaired performance. In another study, this group inhibited the specific neural ensemble controlling the task selectively during the upstates of NREM sleep, which also impaired task performance as well as the ability of the ensemble to develop sparse coding of the task [22•]. Finally, another group showed the same relationship between SOspindle coupling and spindle-nested ripples that indicate cell activation. When they increased sleep spindles by activating parvalbumin interneurons in the reticular nucleus of the thalamus, they increased spindle-ripple coupling in the upstate of SO's and increased memory on both contextual fear memory and novel-object place recognition [30॰]. These data all support the hypothesis that sleep is a time during which synaptic connections are both strengthened and weakened in order to efficiently and accurately encode an animal's environment.

\section{Neural Ensembles Require Offline Processing to Accurately Consolidate New Information}

Optogenetic techniques allow for further exploration into the circuit-level changes underlying disrupted memory consolidation following abnormal sleep dependent activity. Alteration of sleep state activity described in the experiments above broadly results in memory impairment suggesting a relationship between processing during sleep and integrity of memory ensembles. Although they provide invaluable insights, direct manipulation of neural ensemble activity during sleep is required.

\section{Rhythmic Activity like the Theta Oscillation Is Important for Memory Consolidation}

So far, studies suggest that ensembles undergo reshaping during offline processing that can lead to both strengthening and weakening of synaptic connections. The participation of these ensembles in the local rhythmic activity present in sleep appears to be crucial for proper memory formation, in a manner that is highly specific. In an experiment by Kumar et al., optogenetically modulating the activity of adult-born neurons (ABNs) elucidated the role of these cells in consolidating contextual fear memories during REM sleep [21•]. Their study shows that the tuning of $\mathrm{ABNs}$ representing the $\mathrm{CFC}$ memory during REM sleep is critical to the animal's ability to recall this memory the following day. Inactivating the ABN ensemble during REM sleep resulted in poor consolidation of the CFC memory, suggesting the necessity of ABN activity in the DG during REM sleep for memory retention. Silencing ABN activity during NREM sleep following learning did not produce the same effect, indicating a role for REM activity of $\mathrm{ABNs}$ in the processing of fear memories.

\section{Non-ensemble Cells Are Not Involved}

Intriguingly, when $\mathrm{ABNs}$ that were not part of the memory encoding process were randomly stimulated during REM sleep, consolidation was also impaired. This suggests that the integrity of the ensemble active during REM processing is critical to proper consolidation. Similarly, it provides evidence of the causal nature of sleep reactivation for memory consolidation - it suggests a role for the unique population activity of sleep stages in shaping ensemble circuits, which directly contributes to the retention of a memory.

\section{Cortical Ensembles Also Participate in Offline Processing for Memory Consolidation}

The necessity of ensemble reactivation during sleep appears to be critical to brain regions outside of the hippocampus as well. When looking at CFC in the retrosplenial cortex (RSC), researchers found a similarly important role for reactivation in learning and memory [22•]. Isolating stimulation to cells active during a CFC task enabled tightly controlled temporal and spatial modulation of the ensemble presumed to represent the CFC memory trace. To examine the necessity of activation during offline processing to memory integrity, researchers optogenetically stimulated the fear memory ensemble during both anesthesia and natural sleep; ultimately, both conditions produced similar results. This study in particular focused on examining the potential role of sleep-dependent processing in transferring memories to long-term storage. Predictably, animals with activity in their hippocampi blocked $24 \mathrm{~h}$ after learning did not form the proper association between the context and the shock, indicating an improperly consolidated fear memory. This was not the case in the animals that underwent optogenetic stimulation of the RSC memory trace during either anesthesia or sleep right after learning-these mice displayed normal freezing behavior despite the $24 \mathrm{~h}$ later hippocampal block, suggesting that high-frequency activation of the trace ensemble was able to create a hippocampusindependent memory of the fear trace, effectively accelerating the process of systems consolidation. Intriguingly, the optogenetic stimulation protocol was only effective when the animals were in a non-waking state. Stimulation of the fear trace while animals were awake and behaving did not protect against memory impairment following hippocampal 
block, indicating that sleep state activity, or anesthesia plus artificially stimulated activity, is necessary for systems consolidation. Which aspects of sleep are crucial for this process, however, are uncertain, as these experiments did not differentiate between REM or NREM sleep. Additionally, as the researchers note, their stimulation protocol did not maintain the temporal patterns of activity. Given the difference in effectiveness of the optogenetic stimulation between wakefulness and sleep, future research into whether stimulation during only REM or NREM is still effective in inducing systems consolidation would provide more evidence into the role of each sleep stage in long-term memory storage.

Similar work in the visual cortex assessed the effect of offline stimulation of the neural ensemble on memory consolidation [60 ]. Rhythmically activating this trace while an animal was anesthetized biased surrounding neurons toward responding to the same cue during subsequent waking, suggesting a role for offline activity in modifying responsiveness of visual cortical neurons to allow visual discrimination. Inhibition of the cue ensemble during REM and NREM sleep resulted in a generalized behavioral response to all gradients. It will be interesting to observe in future studies what, if anything, distinguishes the roles of REM and NREM sleep in consolidation.

\section{Conclusions and Future Directions}

In summary, applying optogenetic techniques to sleep research has provided an exciting new avenue by which to understand the mechanisms involved in sleep-dependent memory consolidation. We are now able to say with confidence that the negative effects of sleep deprivation on memory consolidation are not solely due to the anxiety- and stressinducing methods of sleep deprivation. Instead, we have begun to determine the specific functions of sleep signatures such as ripples coupled with spindles nested in slow oscillations and theta. The studies we covered here included demonstrations of sleep's involvement in hippocampus-dependent learning such as spatial learning and contextual fear learning, perceptual learning-like visual pattern and texture discrimination learning, motor learning, and even neuroprosthetic learning, the consolidation of all of which are disrupted by specific sleep feature manipulations: delta, theta, spindles, spindleripple coupling, and slow oscillation spindle coupling during REM and non-REM sleep. In fact, using optogenetics to selectively disrupt these features of sleep without altering overall sleep architecture or structure consistently finds learning and memory disruptions, unlike the gross sleep deprivation or disruption studies which have produced more variable results.

Despite these advances, the mysterious nature of sleep still remains, as do challenges to the sleep-memory hypothesis.
For example, we are still unsure of the degree to which experimental sleep studies done in rodents (where the vast majority of our knowledge of sleep's electrophysiology comes from) are applicable to humans. Because of the difficulty of obtaining human intracranial electrophysiological data, for example, we cannot be fully confident that sleep signatures important to rodent learning and memory, such as hippocampal replay or REM sleep theta, are present in human sleep, let alone critical for our sleep-dependent processing. In order to link sleep disturbances to disease and psychiatric illness, more studies will need to be done to demonstrate that sleepdependent consolidation processes in laboratory animal models are analogous to human sleep. Without the ability to target specific neural ensembles in the human brain, as is possible when using optogenetics in animal models, researchers instead use targeted memory reactivation (TMR) as a method to modulate electrophysiological activity during sleep. TMR involves the re-exposure of sensory cues (odor or sound) during sleep that were present during wakeful learning. Unlike the use of light in rodent optogenetic studies, TMR uses cue presentations as a method to selectively reactivate neural ensembles associated with the stimulus. Since the discovery that TMR modulates neural activity in the sleeping brain at the time of cue presentation and improves memory of associated items at subsequent testing by Rasch et al. [61], researchers have sought to elucidate to what extent findings in rodent studies apply to humans. For a full review of recent publications related to this topic, see Cellini and Mednick 2018 [62] and Mankin and Fried 2020 [63]. The use of intracranial recordings to explore sleep in select patient populations as well as new non-invasive technology such as magnetoencephalography in healthy participants will help shed light on some of these questions.

The power of optogenetics as a technique comes from allowing researchers to design experiments that target neurons for modulation in a temporally and spatially specific manner. For sleep research in particular, this means being better equipped to test the underlying mechanisms of sleep without adding confounding variables such as stress or sleep architecture changes into experiments. From just the last few years of research into sleep using optogenetics, we have gathered convincing evidence that without sleep, memories are not processed correctly, largely due to the disturbance of activity patterns underlying sleep signatures such as ripples and spindles. We are beginning to see evidence of sleep-dependent processing occurring in various regions of the brain such as the visual cortex and retrosplenial cortex and, through optogenetic interruption, learning that when the electrophysiology of sleep is disrupted, memory consolidation is impaired as well. The specificity allowed by optogenetic techniques means we are able to narrow down our inquiries to single neuronal ensembles representing a memory trace and observe the impact of sleep-dependent consolidation on that memory alone. 
Acknowledgements Supported by NIMH 60-670. YC was supported by a Cota-Robles Fellowship. RSG was supported by the Integrative Biology and Physiology Eureka Scholarship and T32 NS115753. We thank Rockelle Guthrie for helpful suggestions and for initiating and populating much of the table.

Author Contribution MAF wrote the ms and prepared the figure and citations. YC co-wrote the ms and contributed to the table. RSG made the table and contributed to the ms. GRP co-wrote the ms and edited the figure and table.

\section{Declarations}

Conflicts of Interest The authors declare no competing interests.

Human and Animal Rights and Informed Consent This review article does not report any previously unpublished studies with human or animal subjects performed by any of the authors.

Open Access This article is licensed under a Creative Commons Attribution 4.0 International License, which permits use, sharing, adaptation, distribution and reproduction in any medium or format, as long as you give appropriate credit to the original author(s) and the source, provide a link to the Creative Commons licence, and indicate if changes were made. The images or other third party material in this article are included in the article's Creative Commons licence, unless indicated otherwise in a credit line to the material. If material is not included in the article's Creative Commons licence and your intended use is not permitted by statutory regulation or exceeds the permitted use, you will need to obtain permission directly from the copyright holder. To view a copy of this licence, visit http://creativecommons.org/licenses/by/4.0/.

\section{References}

Papers of particular interest, published recently, have been highlighted as:

- Of importance

- Of major importance

1. Ramanathan L, Gulyani S, Nienhuis R, Siegel JM. Sleep deprivation decreases superoxide dismutase activity in rat hippocampus and brainstem. NeuroReport. 2002;13:1387-90.

2. Gopalakrishnan A, Ji LL, Cirelli C. Sleep Deprivation and cellular responses to oxidative stress. Sleep. 2004;27:27-35. https://doi.org/ 10.1093/sleep/27.1.27.

3. Lima SL, Rattenborg NC, Lesku JA, Amlaner CJ. Sleeping under the risk of predation. Anim Behav. 2005;70:723-36. https://doi.org/ 10.1016/j.anbehav.2005.01.008.

4. Seibt J, Dumoulin MC, Aton SJ, Coleman T, Watson A, Naidoo N, et al. Protein synthesis during sleep consolidates cortical plasticity in vivo. Curr Biol. 2012;22:676-82. https://doi.org/10.1016/j.cub. 2012.02.016.

5. Ramm P, Smith CT. Rates of cerebral protein synthesis are linked to slow wave sleep in the rat. Physiol Behav. 1990;48:749-53. https://doi.org/10.1016/0031-9384(90)90220-X.

6. Vertes RP, Eastman KE. The case against memory consolidation in REM sleep. Behav Brain Sci. 2000;23:867-76. https://doi.org/10. 1017/S0140525X00004003.

7. Youngblood BD, Zhou J, Smagin GN, Ryan DH, Harris RBS. Sleep deprivation by the "flower pot" technique and spatial reference memory. Physiol Behav. 1997;61:249-56. https://doi. org/10.1016/S0031-9384(96)00363-0.

8. Youngblood BD, Smagin GN, Elkins PD, Ryan DH, Harris RBS. The effects of paradoxical sleep deprivation and valine on spatial learning and brain 5-HT Metabolism. Physiol Behav. 1999;67:6439. https://doi.org/10.1016/S0031-9384(99)00120-1.

9. Smith C, Rose GM. Evidence for a paradoxical sleep window for place learning in the Morris water maze. Physiol Behav. 1996;59: 93-7. https://doi.org/10.1016/0031-9384(95)02054-3.

10. Smith C, Conway J, Rose G. Brief paradoxical sleep deprivation impairs reference, but not working, memory in the radial arm maze task. Neurobiol Learn Mem. 1998;69:211-7. https://doi.org/10. 1006/nlme.1997.3809.

11. McDermott CM, LaHoste GJ, Chen C, Musto A, Bazan NG, Magee JC. Sleep deprivation Causes behavioral, synaptic, and membrane excitability alterations in hippocampal neurons. J Neurosci. 2003;23:9687-95. https://doi.org/10.1523/ JNEUROSCI.23-29-09687.2003.

12. McDermott CM, Hardy MN, Bazan NG, Magee JC. Sleep deprivation-induced alterations in excitatory synaptic transmission in the CA1 region of the rat hippocampus. J Physiol. 2006;570: 553-65. https://doi.org/10.1113/jphysiol.2005.093781.

13. Ribeiro S, Mello CV, Velho T, Gardner TJ, Jarvis ED, Pavlides C. Induction of hippocampal long-term potentiation during waking leads to increased extrahippocampal zif-268 expression during ensuing rapid-eye-movement sleep. J Neurosci. 2002;22:10914-23. https://doi.org/10.1523/JNEUROSCI.22-24-10914.2002.

14. Schabus M, Gruber G, Parapatics S, Sauter C, Klösch G, Anderer P, et al. Sleep spindles and their significance for declarative memory consolidation. Sleep. 2004;27:1479-85. https://doi.org/10.1093/ sleep/27.7.1479.

15. Demanuele C, Bartsch U, Baran B, Khan S, Vangel MG, Cox R, et al. Coordination of slow waves with sleep spindles predicts sleepdependent memory consolidation in schizophrenia. Sleep. 2017;40. https://doi.org/10.1093/sleep/zsw013.

16. Mednick S, Nakayama K, Stickgold R. Sleep-dependent learning: a nap is as good as a night. Nat Neurosci. 2003;6:697-8. https://doi. org/10.1038/nn1078.

17. Karni A, Tanne D, Rubenstein BS, Askenasy JJ, Sagi D. Dependence on REM sleep of overnight improvement of a perceptual skill. Science. 1994;265:679-82. https://doi.org/10.1126/ science. 8036518 .

18. Siegel JM. The REM sleep-memory consolidation hypothesis. Science. 2001;294:1058-63. https://doi.org/10.1126/science. 1063049.

19. Vertes RP, Siegel JM. Time for the sleep community to take a critical look at the purported role of sleep in memory processing. Sleep. 2005;28:1228-9. https://doi.org/10.1093/sleep/28.10.1228.

20. Pastrana E. Optogenetics: controlling cell function with light. Nat Methods. 2011;8:24-5. https://doi.org/10.1038/nmeth.f.323.

21. Kumar D, Koyanagi I, Carrier-Ruiz A, Vergara P, Srinivasan S, Sugaya Y, et al. Sparse activity of hippocampal adult-born neurons during REM sleep is necessary for memory consolidation. Neuron. 2020;(107):552, e10-65. https://doi.org/10.1016/j.neuron.2020.05. 008 Adult-born neurons in the dentate gyrus are critical for forming contextual fear memories. REM periods following contextual fear conditioning pare down the activity of these neurons, and silencing them during REM sleep results in unsuccessful fear memory consolidation.

22. de Sousa AF, Cowansage KK, Zutshi I, Cardozo LM, Yoo EJ, Leutgeb S, et al. Optogenetic reactivation of memory ensembles in the retrosplenial cortex induces systems consolidation. Proc Natl Acad Sci. 2019;116:8576-81. https://doi.org/10.1073/pnas. 1818432116 Reactivating neural ensembles outside of the hippocampus is also shown to result in systems consolidation. 
23. Davis CJ, Vanderheyden WM. Optogenetic sleep enhancement improves fear-associated memory processing following trauma exposure in rats. Sci Rep. 2020;10:18025. https://doi.org/10.1038/ s41598-020-75237-9.

24. Izawa S, Chowdhury S, Miyazaki T, Mukai Y, Ono D, Inoue R, et al. REM sleep-active MCH neurons are involved in forgetting hippocampus-dependent memories. Science. 2019;365:1308-13. https://doi.org/10.1126/science.aax9238.

25. Kim J, Gulati T, Ganguly K. Competing roles of slow oscillations and delta waves in memory consolidation versus forgetting. Cell. 2019;179:514-526.e13. https://doi.org/10.1016/j.cell.2019.08.040 Inhibiting the neural ensemble in the motor cortex during the more global slow oscillation upstates impairs memory consolidation, while driving more spindle-slow oscillation upstate coupling through motor neuron ensemble inhibition during the more local delta wave upstates enhances sleepdependent memory motor learning consolidation.

26. Swift KM, Gross BA, Frazer MA, Bauer DS, KJD C, Vazey EM, et al. Abnormal Locus coeruleus sleep activity alters sleep signatures of memory consolidation and impairs place cell stability and spatial memory. Curr Biol. 2018;28:3599-3609.e4. https://doi.org/ 10.1016/j.cub.2018.09.054 Eliminating LC silences during sleep results in unstable spatial representations and performance deficits on a spatial memory task that correlate with delta and theta rhythm amplitude reductions, with curtailment of longer spindles, and with disruptions in spindle-ripple timing that implicate different memory consolidation functions for each sleep state.

27. Lu Y, Zhu ZG, Ma QQ, Su YT, Han Y, Wang X, et al. A critical timewindow for the selective induction of hippocampal memory consolidation by a brief episode of slow-wave sleep. Neurosci Bull. 2018;34: 1091-9. https://doi.org/10.1007/s12264-018-0303-x.

28. Ognjanovski N, Broussard C, Zochowski M, Aton SJ. Hippocampal network oscillations rescue memory consolidation deficits caused by sleep loss. Cereb Cortex. 2018;28:3711-23. https://doi.org/10.1093/cercor/bhy174.

29. Gulati T, Guo L, Ramanathan DS, Bodepudi A, Ganguly K. Neural reactivations during sleep determine network credit assignment. Nat Neurosci. 2017;20:1277-84. https://doi.org/10.1038/nn.4601.

30. Latchoumane CV, Ngo HV, Born J, Shin HS. Thalamic spindles promote memory formation during sleep through triple phaselocking of cortical, thalamic, and hippocampal rhythms. Neuron. 2017;95(2):424-435.e6 https://pubmed.ncbi.nlm.nih.gov/ 28689981/. Timing optogenetic spindle induction to slow oscillation up-phase, along with co-occurring ripples, directly contributes to fear memory consolidation.

31. Boyce R, Glasgow SD, Williams S, Adamantidis A. Causal evidence for the role of REM sleep theta rhythm in contextual memory consolidation. Science. 2016;352:812-6. https://doi.org/10.1126/ science.aad5252.

32. Kovács KA, O’Neill J, Schoenenberger P, Penttonen M, Guerrero DKR, Csicsvari J. Optogenetically Blocking sharp wave ripple events in sleep does not interfere with the formation of stable spatial representation in the CA1 area of the hippocampus. PLoS One. 2016;11:e0164675. https://doi.org/10.1371/journal.pone.0164675.

33. Miyamoto D, Hirai D, Fung CCA, Inutsuka A, Odagwa M, Suzuki T, et al. Top-down cortical input during NREM sleep consolidates perceptual memory. Science. 2016:1315-8 https://science. sciencemag.org/content/352/6291/1315.full.

34. van de Ven GM, Trouche S, McNamara CG, Allen K, Dupret D. Hippocampal offline reactivation consolidates recently formed cell assembly patterns during sharp wave-ripples. Neuron. 2016;92: 968-74. https://doi.org/10.1016/j.neuron.2016.10.020.

35. McNamara CG, Tejero-Cantero Á, Trouche S, Campo-Urriza N, Dupret D. Dopaminergic neurons promote hippocampal reactivation and spatial memory persistence. Nat Neurosci. 2014;17(12):1658-60 https://pubmed.ncbi.nlm.nih.gov/ 25326690/.

36. Rolls A, Colas D, Adamantidis A, Carter M, Lanre-Amos T, Heller $\mathrm{HC}$, et al. Optogenetic disruption of sleep continuity impairs memory consolidation. Proc Natl Acad Sci. 2011;108:13305-10. https:// doi.org/10.1073/pnas.1015633108.

37. Guzman-Marin R, Suntsova N, Methippara M, Greiffenstein R, Szymusiak R, McGinty D. Sleep deprivation suppresses neurogenesis in the adult hippocampus of rats. Eur J Neurosci. 2005;22:2111-6. https://doi.org/10.1111/j.1460-9568.2005.04376. $\mathrm{x}$.

38. Mirescu C, Peters JD, Noiman L, Gould E. Sleep deprivation inhibits adult neurogenesis in the hippocampus by elevating glucocorticoids. Proc Natl Acad Sci. 2006;103:19170-5. https://doi.org/ 10.1073/pnas.0608644103.

39. Bourgin P, Huitrón-Reséndiz S, Spier AD, Fabre V, Morte B, Criado JR, et al. Hypocretin-1 modulates rapid eye movement sleep through activation of locus coeruleus neurons. J Neurosci. 2000;20: 7760-5. https://doi.org/10.1523/JNEUROSCI.20-20-07760.2000.

40. Carter ME, de Lecea L, Adamantidis A. Functional wiring of hypocretin and LC-NE neurons: implications for arousal. Front Behav Neurosci. 2013;7. https://doi.org/10.3389/fnbeh.2013. 00043.

41. España RA, Reis KM, Valentino RJ, Berridge CW. Organization of hypocretin/orexin efferents to locus coeruleus and basal forebrain arousal-related structures. J Comp Neurol. 2005;481:160-78. https://doi.org/10.1002/cne.20369.

42. Palchykova S, Winsky-Sommerer R, Meerlo P, Dürr R, Tobler I. Sleep deprivation impairs object recognition in mice. Neurobiol Learn Mem. 2006;85:263-71. https://doi.org/10.1016/j.nlm.2005. 11.005.

43. Chen L, Tian S, Ke J. Rapid eye movement sleep deprivation disrupts consolidation but not reconsolidation of novel object recognition memory in rats. Neurosci Lett. 2014;563:12-6. https://doi.org/ 10.1016/j.neulet.2014.01.024.

44. Cohen SJ, Munchow AH, Rios LM, Zhang G, Ásgeirsdóttir HN, Stackman RW. The rodent hippocampus is essential for nonspatial object memory. Curr Biol. 2013;23:1685-90. https://doi.org/10. 1016/j.cub.2013.07.002.

45. Corinna K, Dieter R, Nofzinger EA, Bernd F, Josef U, Ruth O'H, et al. Memory before and after sleep in patients with moderate obstructive sleep apnea. J Clin Sleep Med. 2009;05:540-8. https://doi.org/10.5664/jcsm.27655.

46. Aston-Jones G, Bloom FE. Activity of norepinephrine-containing locus coeruleus neurons in behaving rats anticipates fluctuations in the sleep-waking cycle. J Neurosci. 1981;1:876-86. https://doi.org/ 10.1523/JNEUROSCI.01-08-00876.1981.

47. Wilson MA, McNaughton BL. Reactivation of hippocampal ensemble memories during sleep. Science. 1994;265:676-9. https:// doi.org/10.1126/science.8036517.

48. Kudrimoti HS, Barnes CA, McNaughton BL. Reactivation of hippocampal cell assemblies: effects of behavioral state, experience, and EEG dynamics. J Neurosci. 1999;19:4090-101. https://doi.org/ 10.1523/JNEUROSCI.19-10-04090.1999.

49. Pavlides C, Winson J. Influences of hippocampal place cell firing in the awake state on the activity of these cells during subsequent sleep episodes. J Neurosci. 1989;9:2907-18. https://doi.org/10.1523/ JNEUROSCI.09-08-02907.1989.

50. Girardeau G, Benchenane K, Wiener SI, Buzsáki G, Zugaro MB. Selective suppression of hippocampal ripples impairs spatial memory. Nat Neurosci. 2009;12:1222-3. https://doi.org/10.1038/nn. 2384.

51. Ego-Stengel V, Wilson MA. Disruption of ripple-associated hippocampal activity during rest impairs spatial learning in the rat. Hippocampus. 2010;20:1-10. https://doi.org/10.1002/hipo.20707. 
52. Li W, Ma L, Yang G, Gan W-B. REM sleep selectively prunes and maintains new synapses in development and learning. Nat Neurosci. 2017;20:427-37. https://doi.org/10.1038/nn.4479.

53. Zhou Y, Lai CSW, Bai Y, Li W, Zhao R, Yang G, et al. REM sleep promotes experience-dependent dendritic spine elimination in the mouse cortex. Nat Commun. 2020;11:4819. https://doi.org/10. 1038/s41467-020-18592-5.

54. Fernández-Ruiz A, Oliva A, Nagy GA, Maurer AP, Berényi A, Buzsáki G. Entorhinal-CA3 dual-input control of spike timing in the hippocampus by theta-gamma coupling. Neuron. 2017;93: 1213-1226.e5. https://doi.org/10.1016/j.neuron.2017.02.017.

55. Dragoi G, Buzsáki G. Temporal encoding of place sequences by hippocampal cell assemblies. Neuron. 2006;50:145-57. https://doi. org/10.1016/j.neuron.2006.02.023.

56. Foster DJ, Wilson MA. Hippocampal theta sequences. Hippocampus. 2007;17:1093-9. https://doi.org/10.1002/hipo. 20345.

57. Gupta AS, van der Meer MAA, Touretzky DS, Redish AD. Segmentation of spatial experience by hippocampal theta sequences. Nat Neurosci. 2012;15:1032-9. https://doi.org/10.1038/ nn.3138.

58. Poe GR, Nitz DA, McNaughton BL, Barnes CA. Experiencedependent phase-reversal of hippocampal neuron firing during REM sleep. Brain Res. 2000;855:176-80. https://doi.org/10.1016/ S0006-8993(99)02310-0.

59. Poe GR. Sleep Is for Forgetting. J Neurosci. 2017;37:464-73. https://doi.org/10.1523/JNEUROSCI.0820-16.2017.
60. Clawson BC, Pickup EJ, Enseng A, Geneseo L, Shaver J, Gonzalez-Amoretti J, et al. Causal role for sleep-dependent reactivation of learning-activated sensory ensembles for fear memory consolidation. Nat Commun. 2021;12:1200. https://doi.org/10. 1038/s41467-021-21471-2 Visual cue-responsive neurons are selectively reactivated during post-fear conditioning sleep. When these ensembles are inhibited, fear memory consolidation is disrupted, suggesting the selective reactivation of sensory information during sleep is necessary for memory consolidation.

61. Rasch B, Büchel C, Gais S, Born J. Odor cues during slow-wave sleep prompt declarative memory consolidation. Science. 2007;315:1426-9 https://pubmed.ncbi.nlm.nih.gov/17347444/.

62. Cellini N, Mednick SC. Stimulating the sleeping brain: current approaches to modulating memory-related sleep physiology. J Neurosci Methods. 2019;316:125-36 https://pubmed.ncbi.nlm. nih.gov/30452977/.

63. Mankin EA, Fried I. Modulation of human memory by deep brain stimulation of the entorhinal-hippocampal circuitry. Neuron. 2020;106(2):218-35. https://doi.org/10.1016/j.neuron.2020.02. 024.

Publisher's Note Springer Nature remains neutral with regard to jurisdictional claims in published maps and institutional affiliations. 